\title{
Opportunistic Visualization with iVoLVER
}

\author{
Gonzalo Gabriel Méndez \\ University of St Andrews, UK \\ ggm@st-andrews.ac.uk
}

\author{
Miguel A. Nacenta \\ University of St Andrews, UK \\ mans@st-andrews.ac.uk
}

\begin{abstract}
Proposed as "data analysis anywhere, anytime, from anything", Opportunistic Information Visualization (OpportuVis) [1] seeks to provide analytical support in scenarios where the data of interest is not explicitly available and has to be retrieved from digital artifacts that are not traditionally used as data sources. Examples include raster images, web pages, vector files, and photographs. This showpiece presents how iVoLVER, the Interactive Visual Language for Visualization Extraction and Reconstruction, provides support in such settings. We briefly describe the overall construction approach of the tool in scenarios where different digital artifacts are used to compose interactive visuals. All of this becomes possible by using the data extraction capabilities of iVoLVER together with the elements of its visual language.
\end{abstract}

\section{INTRODUCTION}

In a world of ever-growing information we are surrounded by data that exists in many forms. Ranging from photographs to web pages, we are constantly exposed to data encoded within a wide variety of digital artifacts. In spontaneous, informal data analyses, some of these artifacts could provide useful input for the construction of visualizations. The way they contain information, however, prevents their immediate use and manipulation in the vast majority of current visualization authoring systems. This is partly because traditional visualization tools mostly work on raw data structured and organized in specific formats (e.g., databases, Excel tables, or comma-separated files); but also because the construction of visualizations normally requires specific input and output devices (such us displays, mouse, and keyboard) that might not be available in opportunistic settings.

iVoLVER $^{1}$, the Interactive Visual Language for Visualization Extraction and Reconstruction [2], is a novel web-based pen and touch tool that enables the creation of visualizations from multiple non-traditional data sources (e.g., photographs, bitmap charts, web pages, vector images). This showpiece proposes an interactive demonstration to present our ongoing work on the system. The demonstration is addressed to a broad audience: from possible users of the tool to experts in visual programming languages and interaction that are interested in its features, design, and implementation. Because of its visual nature and its interactive user-driven construction approach, iVoLVER aligns with the research topics and interests promoted within the VL/HCC conference. We envision this demonstration as a way to

\footnotetext{
${ }^{1}$ http://ivolver.cs.st-andrews.ac.uk
}

stimulate discussions on how the VL/HCC community can further contribute to support visualization in opportunistic settings, where raw data-as traditionally understood-is not explicitly available to users.

\section{IVOLVER CONSTRUCTIONS}

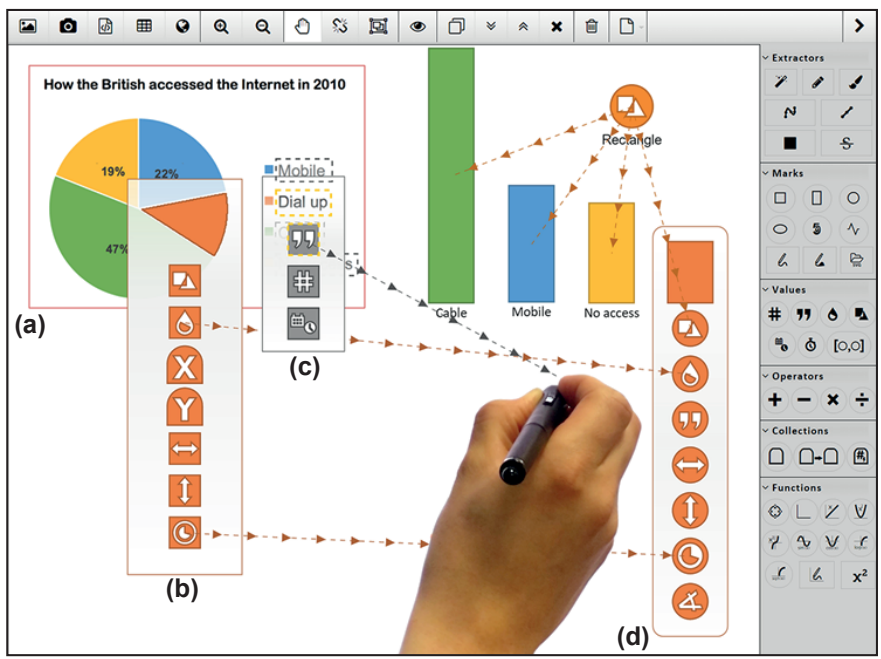

Fig. 1. An image of a pie chart (a) is overlaid with several iVoLVER extractors. The data provided by a color (b) and a text (c) extractors is used to configure the appearance of four rectangular marks that compose a bar representation of the original visualization. The connections show the dataflow between the extractors and the visual properties of the orange mark (d).

iVoLVER constructions are implemented by connecting components of its visual language within a zoomable infinite canvas where different types of digital artifacts can be imported (see Fig. 1). Graphical interfaces called Extractors provide data extraction functionalities. Extractors execute specialized routines (such as image processing and text recognition) depending on their type. Users drive the construction process via simple gestures and interactions. For example, to extract the data of a color region, the user activates the respective extraction mode (via the right menu of the interface) and double-taps on top of the corresponding imported image. This triggers the execution of a flood fill algorithm that detects the colored area underneath the user's input point. The newly created extractor is then overlaid on top of the image.

Other types of digital artifacts do not require specialized extraction routines. Web pages, for instance, can be opened within the system in a browser-like interface from which 

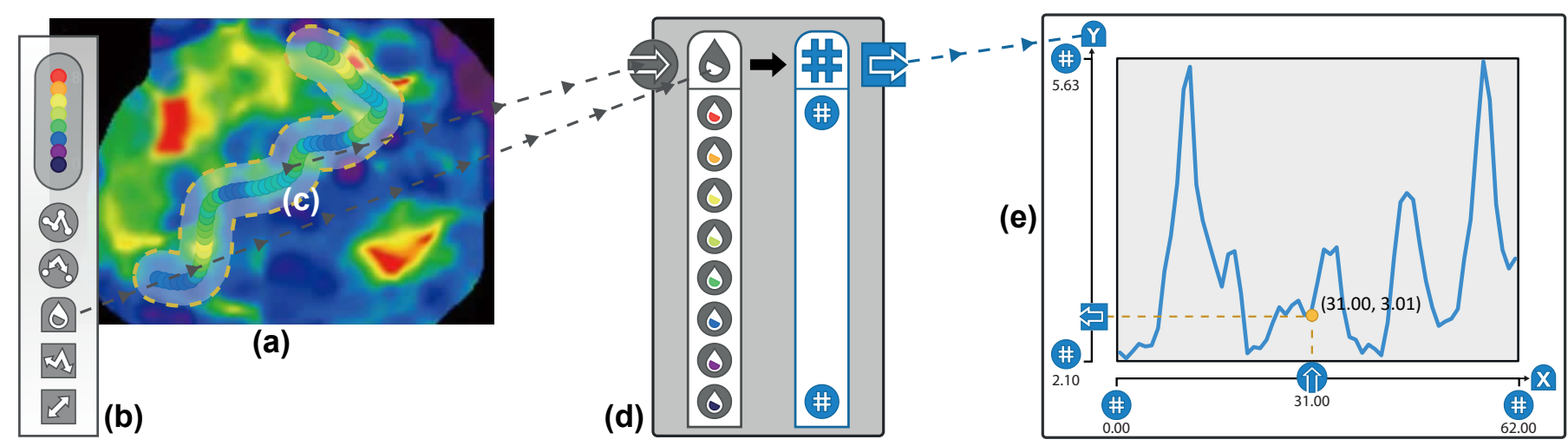

Fig. 2. Color data is extracted from an image of a magnetic resonance to generate the profile of a user defined path. See description in Section II.

information (e.g. text, images) can be directly dragged onto the canvas (see Fig. 3). This type of extraction exploits the DOM structure of the HTML documents, which also allows for the identification of the semantics behind the extracted data (e.g., when dragged onto the canvas, HTML lists are represented as iVoLVER Collections).

Most of iVoLVER elements can be displayed in two possible states: compressed and expanded. When expanded, objects expose their visual properties, each of which stores a value of a specific type. Inspired in the concepts proposed by Bertin [3], visual properties can be used to: 1) define the visual appearance of certain objects, 2) expose values to other elements in the canvas, or 3) to configure the data extraction parameters used by extractors. A color-based extractor, for instance, has a shape property, a color one, three numeric values (width, height and area of the extracted region), and two collections of numeric values that contain the $x$ and $y$ coordinates of the points that define its shape. Connections

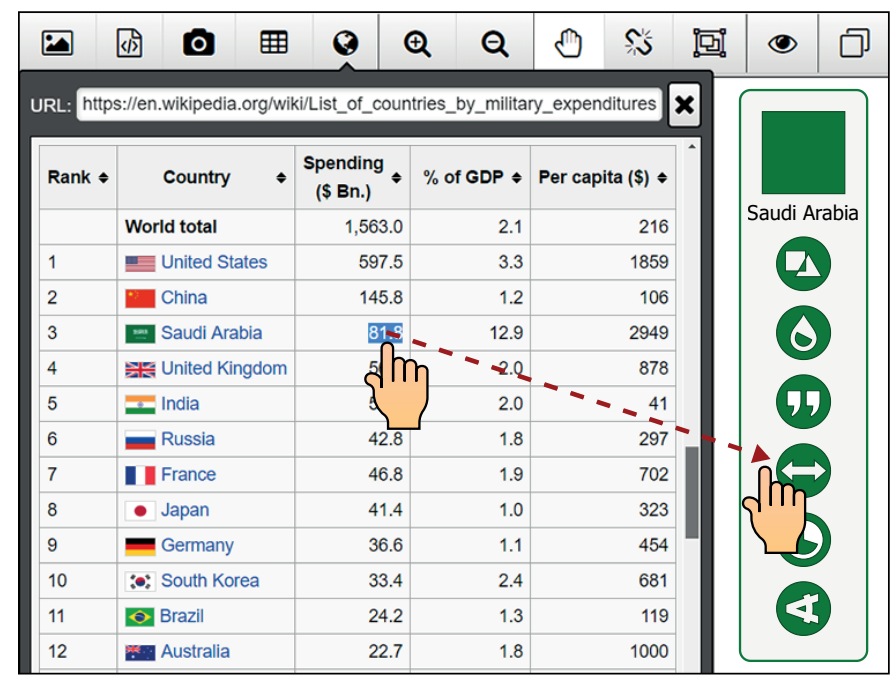

Fig. 3. Web pages can be opened within the iVoLVER interface and their data can be directly dragged onto the canvas or used to modify visual properties. created between visual properties and other components of a visualization indicate the flow of information and define the interactive behavior of the construction.

Figure 2 shows a color map image (adapted from [4]) resulting from a magnetic resonance procedure (a). Two colors samplers-extractors that collect color data along a userdefined path-have been overlaid on top of the image (b, c). The extracted color data is used to build a numeric signal profile of the path defined by the second color sampler (c). The transformation from colors to numbers takes place in a mapper (d), which represents the legend of the image and visually establishes the corresponding relation between colors and numbers. The resulting numeric values are finally visualized with a function element (e). This scenario exemplifies the creation of a visualization with a specific purpose from spatial coordinates that are then translated into numbers.

\section{CONCLUSION}

This showcase presents iVoLVER, a tool that visually supports analysis of data in opportunistic scenarios. iVoLVER provides data extraction capabilities to retrieve data encoded in a diverse set of digital artifacts. We believe iVoLVER can motivate fruitful discussions within the VL/HCC community on the design and implementation of novel interfaces to support OpportuVis.

\section{REFERENCES}

[1] G. G. Méndez, "Tools for opportunistic information visualization: Visual analysis with non-traditional data sources," in Symposium on Visual Languages and Human-Centric Computing (VLlHCC). IEEE, 2016.

[2] G. G. Méndez, M. A. Nacenta, and S. Vandenheste, "ivolver: Interactive visual language for visualization extraction and reconstruction," in Proceedings of the 2016 CHI Conference on Human Factors in Computing Systems, ser. CHI '16. New York, NY, USA: ACM, 2016, pp. 4073-4085. [Online]. Available: http://doi.acm.org/10.1145/2858036. 2858435

[3] J. Bertin, Semiology of Graphics: Diagrams, Networks, Maps. Redlands, Calif: ESRI Press, Oct. 2011.

[4] T. Hennedige and S. K. Venkatesh, "Advances in computed tomography and magnetic resonance imaging of hepatocellular carcinoma," World journal of gastroenterology, vol. 22, no. 1, p. 205, 2016. 